\title{
Could Improvement in Subjective Well-being Relieve Gastrointestinal Symptoms in Patients With Irritable Bowel Syndrome?
}

\author{
Seung-Ho Jang ${ }^{1}$ and Yong Sung Kim ${ }^{2 *}$ \\ ${ }^{1}$ Department of Psychiatry, Wonkwang Digestive Research Institute, School of Medicine, Wonkwang University, Iksan, Jeollabuk-do, Korea; \\ and ${ }^{2}$ Department of Gastroenterology, Wonkwnag Microbiota-Brain-Gut Axis Research Center, Wonkwang University Sanbon Hospital, Gunpo, \\ Gyeonggi-do, Korea
}

Article: Connecting our gut feeling and how our gut feels: the role of well-being attributes in irritable bowel syndrome Farhadi A, Banton D, Keefer L

(J Neurogastroenterol Motil 2018;24:289-298)

Irritable bowel syndrome (IBS) is the most representative disease that can be explained by the brain-gut axis. Psychiatric comorbidity plays an important role in the pathogenesis of IBS, which is frequently accompanied by depression and anxiety. A recent metaanalysis revealed that the prevalence of depression and anxiety was higher in patients with IBS, regardless of the IBS subtype, than in healthy people. ${ }^{1}$ Stress and sexual, physical, and emotional abuses are also important factors in the onset of IBS. ${ }^{2}$

While studies on the above-mentioned abnormal psychology in IBS have been actively conducted, there is little interest in positive psychology. In this issue of the Journal of Neurogastroenterology and Motility, Farhadi et al ${ }^{3}$ demonstrated that subjective well-being (SWB) and several well-being attributes are negatively associated with gastrointestinal symptoms and IBS. Of interest, this relationship is independent of anxiety and depression. To the best of knowledge, this is the first study to investigate the role of SWB, a positive psychology in patients with IBS. SWB, which may be an unfamiliar concept to gastroenterologists, is how people evaluate their lives emotionally and cognitively. ${ }^{4}$ Someone with a high level of SBW can be simply thought of as one who is very happy. Despite the high prevalence of IBS, its pathogenesis is not clear and no effective treatments have been developed until now. Therefore, a new approach is needed to overcome this problem. In this respect, this study suggests that SWB may be one of the pathogenic mechanisms of IBS. Some of the known risk factors for IBS could be understood in the relation with SWB. For example, marital status is an important factor for higher SWB, so married persons' SWB is higher than that of those who are single or cohabiting. ${ }^{4}$ Similarly, single/divorced or widowed marital status has been known as an independent risk factor for higher medical utilization or overlap with functional dyspepsia in patients with IBS. ${ }^{5,6} \mathrm{We}$ can also speculate that perceived stigmata commonly found in patients with IBS may decrease SWB. ${ }^{2,7}$

Several things should be considered before adopting SWB in clinical research and practice. SWB is a complex domain that combines various well-being attributes, so the SWB score may vary depending on the measurement methods and emotional state or situation at the point of measurement. ${ }^{4}$ SWB is closely related

Received: March 8, 2018 Revised: None Accepted: March 13, 2018

(.) This is an Open Access article distributed under the terms of the Creative Commons Attribution Non-Commercial License (http://creativecommons. org/licenses/by-nc/4.0) which permits unrestricted non-commercial use, distribution, and reproduction in any medium, provided the original work is properly cited.

*Correspondence: Yong Sung Kim, MD, PhD

Department of Gastroenterology, Wonkwang University Sanbon Hospital, 321 Sanbon-ro, Gunpo, Gyeonggi-do 15865, Korea Tel: +82-31-390-2973, Fax: +82-31-390-2244, E-mail: wms89@hanmail.net 
to personalities such as extroversion or neuroticism, and to some genetic predisposition. ${ }^{8}$ Finally, it should be noted that SWB is influenced by gender and cultural background. ${ }^{9}$ Thus, in the case of marriage mentioned above, the effect size of marital status on SWB or IBS symptom could be different between men and women, or Westerners and Easterners. ${ }^{10}$

Although it is a preliminary online survey with many limitations and must be validated for real patients with IBS in clinical practice, this study has another important implication that suggests the possibility of SBW as a new therapeutic target. Therefore, after studying the role of SWB in patients with IBS, whether IBS symptoms can be alleviated by improving low SWB level should be elucidated in future studies. At least the SWB concept can provide a rationale for current treatment methods such as mindfulness, exercise, and increased job satisfaction and familial support for IBS patients.

Like the psychiatrist Hector in the movie Hector and the Search for Happiness, for some cases, the gastroenterologist may need to find happiness together with their patients with IBS for better treatment in addition to medication.

Financial support: This work was supported by Wonkwang University 2017.

\section{Conflicts of interest: None.}

Author contributions: Seung-Ho Jang drafted and revised the manuscript; and Yong Sung Kim drafted, revised, and performed the final approval.

\section{References}

1. Lee C, Doo E, Choi JM, et al. The increased level of depression and anxiety in irritable bowel syndrome patients compared with healthy controls: systematic review and meta-analysis. J Neurogastroenterol Motil 2017;23:349-362.

2. Van Oudenhove L, Crowell MD, Drossman DA, et al. Biopsychosocial aspects of functional gastrointestinal disorders. Gastroenterology 2016;150:1355-1367, e2.

3. Farhadi A, Banton D, Keefer L. Connecting our gut feeling and how our gut feels: the role of well-being attributes in irritable bowel syndrome. J Neurogastroenterol Motil 2018;24:289-298.

4. Diener E, Oishi S, Lucas RE. Personality, culture, and subjective wellbeing: emotional and cognitive evaluations of life. Annu Rev Psychol 2003;54:403-425.

5. Gudleski GD, Satchidanand N, Dunlap LJ, et al. Predictors of medical and mental health care use in patients with irritable bowel syndrome in the United States. Behav Res Ther 2017;88:65-75.

6. Choi YJ, Kim N, Yoon H, et al. Overlap between irritable bowel syndrome and functional dyspepsia including subtype analyses. J Gastroenterol Hepatol 2017;32:1553-1561.

7. Jia X, Liu X, Shi B. Perceived discrimination and subjective well-being in Chinese migrant adolescents: collective and personal self-esteem as mediators. Front Psychol 2017;8:1213.

8. Nes RB, Røysamb E, Harris JR, Czajkowski N, Tambs K. Mates and marriage matter: genetic and environmental influences on subjective wellbeing across marital status. Twin Res Hum Genet 2010;13:312-321.

9. Derdikman-Eiron R, Indredavik MS, Bratberg GH, Taraldsen G, Bakken IJ, Colton M. Gender differences in subjective well-being, selfesteem and psychosocial functioning in adolescents with symptoms of anxiety and depression: findings from the Nord-Trondelag Health Study. Scand J Psychol 2011;52:261-267.

10. Fang X, Francisconi CF, Fukudo S, et al. Multicultural aspects in functional gastrointestinal disorders (FGIDs). Gastroenterology 2016;150:1344-1354, e2. 